\title{
Dendritic Macromers as In Situ Polymerizing Biomaterials for Securing Cataract Incisions
}

Michel Wathier, Pil J. Jung, ${ }^{\dagger}$ Michael A. Carnahan, ${ }^{\dagger}$ Terry Kim, ${ }^{\dagger}$ and Mark W. Grinstaff

Departments of Biomedical Engineering and Chemistry, Metcalf Center for Science and Engineering, Boston University, Boston, MA 02215, USA. 'Department of Ophthalmology, Duke University Medical Center, Durham NC 27710.

\section{Experimental section}

Material. All solvents were dried and freshly distilled prior to use $\left(\mathrm{CH}_{2} \mathrm{Cl}_{2}\right.$ with $\mathrm{CaH}_{2}$ and $\mathrm{MeOH}$ with $\mathrm{Na}$ ) or were purchased from Acros (DMF). All chemicals were purchased from Aldrich, Acros, Shearwater or CHEM IMPEX as highest purity grade and used without further purification. All reactions were performed under nitrogen atmosphere. NMR spectra were recorded on a Varian INOVA spectrometer (for ${ }^{1} \mathrm{H}$ and ${ }^{13} \mathrm{C}$ at 400 and $100.6 \mathrm{MHz}$, respectively). Chemical ionization mass spectra were obtained on a Hewlett-Packard HP 5988A spectrometer using $\mathrm{NH}_{3}$. Fast atom bombardment mass spectra (FABMS) were obtained on a JEOL JMS-SX102A spectrometer using a 3-nitrobenzyl alcohol matrix. Elemental analysis was obtained from Atlantic Microlab, Inc. A TA Instruments RA 1000 was used for the rheological measurements. DPTS $=4$-(dimethylamino)pyridinium 4-toluenesulfate, $\mathrm{DCC}=$ dycyclohexylcarbodiimide, $\mathrm{DMF}=N, N$-dimethylformamide, $\mathrm{DCU}=1,3$ dicyclohexylurea, $\mathrm{Pd} / \mathrm{C}=10 \%$ palladium on activated carbon, $\mathrm{PFP}=2,3,4,5,6$ pentafluorophenol, DIEA = diisopropylethyl amine, HOBT $=$ hydroxybenzotriazol, TFA = trifluoroacetiqueacid, $\mathrm{Z}=$ benzyloxycarbonyl, Boc = terbutyloxycarbonyl, Isopr = isopropylydene. The buffer $\mathrm{pH}=7.4$ was prepared from HEPES $(100 \mathrm{mM})$ with $\mathrm{NaOH}$ $1 \mathrm{~N}$.

\section{Synthesis of ZLys(Z)OPFP}

DCC (5.45 g, $26 \mathrm{mmol})$ in $\mathrm{CH}_{2} \mathrm{Cl}_{2}(20 \mathrm{~mL})$ was added in five portions over 10 minutes to a solution of ZLys(Z)OH (10 g, $24 \mathrm{mmol})$ and PFP (4.49 g, $26 \mathrm{mmol})$ in freshly distilled $\mathrm{CH}_{2} \mathrm{Cl}_{2}(40 \mathrm{~mL})$. The reaction mixture was stirred under $\mathrm{N}_{2}$ at $25{ }^{\circ} \mathrm{C}$ for $2 \mathrm{~h}$, filtered to remove the insoluble DCU, concentrated to $\sim 20 \mathrm{~mL}$ under reduced pressure, and then stored at $4{ }^{\circ} \mathrm{C}$ for $2 \mathrm{~h}$. An additional filtration removed further urea, and the solution was then diluted with hexane $(25 \mathrm{~mL})$ and stored at $4{ }^{\circ} \mathrm{C}$ for $4 \mathrm{~h}$. The resultant white precipitate was collected by filtration, washed with $\mathrm{CH}_{2} \mathrm{Cl}_{2} /$ hexane $(1: 2,3 \times 5 \mathrm{~mL})$, and dried under vacuum; yield $13.37 \mathrm{~g}(98 \%) .{ }^{1} \mathrm{H}$ NMR $\left(\mathrm{CDCl}_{3}\right): \delta 1.46\left(\mathrm{~m}, 2, \mathrm{CH}_{2}-\mathrm{CH}_{2}\right)$; 1.54 (m, 2, $\left.\mathrm{CH}_{2}-\mathrm{CH}_{2}\right) ; 1.84$ (m, 1, $\left.\mathrm{CH}_{2}-\mathrm{CH}\right) ; 2.00$ (m, 1, $\left.\mathrm{CH}_{2}-\mathrm{CH}\right) ; 3.19$ (m, 2, $\left.\mathrm{CH}_{2}-\mathrm{NH}\right)$; 4.67 (m, 1, $\left.\mathrm{CH}_{2}-\mathrm{CH}\right) ; 4.8$ (m, 1, NH); 5.03 (m, 2, $\left.\mathrm{CH}_{2}-\mathrm{O}\right) ; 5.11$ (s, 2, $\left.\mathrm{CH}_{2}-\mathrm{O}\right) ; 5.54$ (m, 1, $\mathrm{NH}) ; 7.3(\mathrm{~m}, 10$, arom $\mathrm{CH}) .{ }^{13} \mathrm{C} \mathrm{NMR}\left(\mathrm{CDCl}_{3}\right): \delta 22.63\left(\mathrm{CH}_{2}\right) ; 30.06\left(\mathrm{CH}_{2}\right) ; 32.10$ $\left(\mathrm{CH}_{2}\right) ; 40.72\left(\mathrm{CH}_{2}-\mathrm{NH}\right) ; 54.33(\mathrm{CH}) ; 67.44$ and $68.04\left(\mathrm{CH}_{2}-\mathrm{O}\right) ; 128.78-129.22(\mathrm{CH}$ arom); 136.61 and 137.13 (C arom); 156.61-157.38 (CO-O-NH); 169.48 (CO ester). ${ }^{19} \mathrm{~F}$ 
NMR $\left(\mathrm{CDCl}_{3}\right): \delta-162.26(\mathrm{t}, 2, \mathrm{C} F) ;-157.60(\mathrm{t}, 1, \mathrm{CF}) ;-152.72(\mathrm{~d}, 2, \mathrm{C} F)$. FAB MS: $581.7 \mathrm{~m} / \mathrm{z}\left(\mathrm{MH}^{+}\right)$(theory: $580.5 \mathrm{~m} / \mathrm{z}$ $\left(\mathrm{M}^{+}\right)$). Elemental analysis: (theory: $\mathrm{C}$, 57.93; H, 4.34) found $\mathrm{C}, 58.12 ; \mathrm{H}, 4.40$.

\section{Synthesis of IsoCys(Boc)OH}

IsoCys(Boc)OH was Prepared following a published paper (D. S. Kemp, R. I. Carey, J. Org. Chem., 1989, 54, 3640). FAB MS: $260.1 \mathrm{~m} / \mathrm{z}\left(\mathrm{MH}^{-}\right)$(theory: $261.1 \mathrm{~m} / \mathrm{z}$ $\left.\left(\mathrm{M}^{+}\right)\right)$.

\section{Synthesis of IsoCys(Boc)OPFP}

IsoCys(Boc)OPFP was prepared in a similar manner as ZLys(Z)OPFP starting from IsoCys(Boc)OH in $95 \%$ yield. ${ }^{1} \mathrm{H}$ NMR $\left(\mathrm{CDCl}_{3}\right): \delta 1.43(\mathrm{~s}, 6$, Boc $\mathrm{CH} 3)$; 1.49 (s, 3, Boc $\left.\mathrm{CH}_{3}\right) ; 1.81$ (s, 3, Isopr $\left.\mathrm{CH}_{3}\right) ; 1.87$ (s, 3, Isopr $\left.\mathrm{CH}_{3}\right) ; 3.24$ (d-d, 1, $\left.\mathrm{CH}_{2}\right) ; 3.43$ (d-d, 1, $\left.\mathrm{CH}_{2}\right) ; 5.14(\mathrm{~d}, 1, \mathrm{CH})$.

${ }^{13} \mathrm{C} \mathrm{NMR}\left(\mathrm{CDCl}_{3}\right): \delta$ 28.53-30.94 $\left(\mathrm{CH}_{3}\right)$; $31.43\left(\mathrm{CH}_{2}\right) ; 65.92$ and $66.60(\mathrm{CH}) ; 70.97$ and $72.54\left(\mathrm{C}-\mathrm{CH}_{3}\right) ; 81.62$ and $82.38(\mathrm{C}$ -
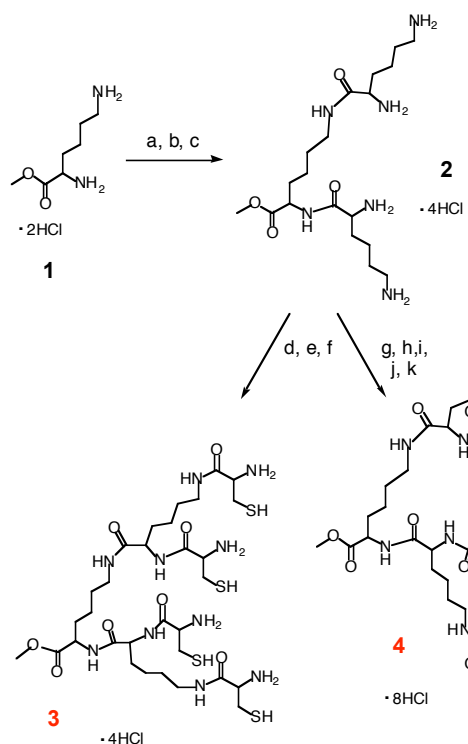

Reactants and conditions: a) Z-Lys(Z)-OPFP, DMF, DIEA, HOBT, $\mathrm{CH}_{2} \mathrm{Cl}_{2}, 25^{\circ} \mathrm{C}$ for $24 \mathrm{~h}, 98 \%$ yield; b) $\mathrm{H}_{2} / \mathrm{Pd} / \mathrm{C}, \mathrm{MeOH}, 6 \mathrm{~h}, 99 \%$ yield; c) $\mathrm{HCl} 1 \mathrm{~N}, 25^{\circ} \mathrm{C}$ for $5 \mathrm{~min}, 99 \%$ yield; d) iso-Cys(Boc)-OPFP, DMF, $\mathrm{CH}_{2} \mathrm{Cl}_{2}$, DIEA, HOBT, $25^{\circ} \mathrm{C}$ for $24 \mathrm{~h}, 96 \%$ yield e) TFA $17 \%$ in $\mathrm{CH}_{2} \mathrm{Cl}_{2}, 25^{\circ} \mathrm{C}$ for $2 \mathrm{~h}, 97 \%$ yield; f) $\mathrm{HCl} 1 \mathrm{~N} / \mathrm{MeOH} 50 / 50,25^{\circ} \mathrm{C}$ for 4 h, $92 \%$ yield; g) Boc-Lys(Boc)-OPFP, DMF, $\mathrm{CH}_{2} \mathrm{Cl}_{2}$, DIEA, HOBT, $25^{\circ} \mathrm{C}$ for $24 \mathrm{~h}$, $65 \%$ yield; h) TFA $15 \%$ in $\mathrm{CH}_{2} \mathrm{Cl}_{2}, 25^{\circ} \mathrm{C}$ for $1 \mathrm{~h}, 99 \%$ yield. i) iso-Cys(Boc)-OPFP DMF, $\mathrm{CH}_{2} \mathrm{Cl}_{2}$, DIEA, $\mathrm{HOBT}, 25^{\circ} \mathrm{C}$ for $24 \mathrm{~h}, 96 \%$ yield; j) TFA $17 \%$ in $\mathrm{CH}_{2} \mathrm{Cl}_{2}, 25^{\circ} \mathrm{C}$ for $2 \mathrm{~h}, 97 \%$ yield; $\mathrm{k}$ ) $\mathrm{HCl} 1 \mathrm{~N} / \mathrm{MeOH} 50 / 50,25^{\circ} \mathrm{C}$ for $4 \mathrm{~h}, 99 \%$ yield;

Scheme S1. Synthesis of dendrons 3 and 4 $\left.\left(\mathrm{CH}_{3}\right)_{3}\right) ; 128.68-129.01$ ( $\mathrm{CH}$ arom $)$; 136.51 and $137.10\left(\mathrm{C}\right.$ arom); $152.00(\mathrm{CO}-\mathrm{O}-\mathrm{NH}) ; 169.39$ (CO ester). ${ }^{19} \mathrm{~F}$ NMR $\left(\mathrm{CDCl}_{3}\right)$ : $\delta-162.24(\mathrm{t}, 2, \mathrm{CF}) ;-157.70(\mathrm{t}, 1, \mathrm{CF}) ;-152.94(\mathrm{~d}, 2, \mathrm{CF})$. GC MS: $445.0 \mathrm{~m} / \mathrm{z}(\mathrm{M}+$ $\mathrm{NH}_{4}^{+}$) (theory: $427.0 \mathrm{~m} / z\left(\mathrm{M}^{+}\right)$). Elemental analysis: (theory: C, 47.77; H, 4.25; N, 3.28; $\mathrm{S}, 7.50$ ) found $\mathrm{C}, 47.74 ; \mathrm{H}, 4.19 ; \mathrm{N}, 3.35 ; \mathrm{S}, 7.48$.

\section{Synthesis of ZLys(Z)Lys(ZLys(Z))OMe}

LysOMe $2 \mathrm{HCl}(1.43 \mathrm{~g}, 6 \mathrm{mmol})$ was dissolved in DMF (45 mL) and DIEA (2.35 g, 18 mmol), and HOBT (2.25 g, $14 \mathrm{mmol})$ were then added. After 5 minutes ZLys(Z)OPFP (12.5 g, $21 \mathrm{mmol})$ in $\mathrm{CH}_{2} \mathrm{Cl}_{2}(30 \mathrm{~mL})$ was added at $0{ }^{\circ} \mathrm{C}$ for $10 \mathrm{~min}$. The mixture was stirred for $24 \mathrm{~h}$ at $\mathrm{RT}$ under $\mathrm{N}_{2}$. After concentration under vacuum the mixture was dissolved again in $\mathrm{CH}_{2} \mathrm{Cl}_{2}(50 \mathrm{~mL})$ and washed with $\mathrm{NaHCO}_{3}(2 \times 150 \mathrm{~mL})$, water $(2 \times 150$ $\mathrm{mL}$ ) and dried over $\mathrm{Na}_{2} \mathrm{SO}_{4}$. The solvent was removed, and the mixture was precipited in ether to afford a pure white compound $5.72 \mathrm{~g}(98 \%) .{ }^{1} \mathrm{H} \mathrm{NMR}\left(\mathrm{CDCl}_{3}\right): \delta 1.35-1.79(\mathrm{~m}$, $\left.18, \mathrm{CH}_{2}-\mathrm{CH}_{2}\right) ; 2.87$ (m, 1, $\left.\mathrm{CH}_{2}-\mathrm{NH}\right) ; 3.13$ (m, 4, $\left.\mathrm{CH}_{2}-\mathrm{NH}\right) ; 3.40$ (m, 1, $\left.\mathrm{CH}_{2}-\mathrm{NH}\right) ; 3.63$ (s, 3, $\left.\mathrm{CH}_{3}\right) ; 4.16(\mathrm{~m}, 1, \mathrm{CH}-\mathrm{NH}) ; 4.34$ (m, 1, CH-NH); 4.38 (m, 1, CH-NH); 4.88-5.02 (4 x s, 8, $\left.\mathrm{CH}_{2}-\mathrm{O}\right) ; 5.13\left(\mathrm{~m}, 1, \mathrm{CH}_{2}-\mathrm{NH}\right) ; 5.28\left(\mathrm{~m}, 1, \mathrm{CH}_{2}-\mathrm{NH}\right) ; 5.94(\mathrm{~d}, 1, \mathrm{CH}-\mathrm{NH}) ; 6.25$ (d, 1, $\mathrm{CH}-\mathrm{NH}) ; 6.88\left(\mathrm{~m}, 1, \mathrm{CH}_{2}-\mathrm{NH}\right) ; 7.19-7.27(\mathrm{~m}, 20$, arom $\mathrm{CH}) .7 .43(\mathrm{~d}, 1, \mathrm{CH}-\mathrm{NH}) .{ }^{13} \mathrm{C}$ NMR $\left(\mathrm{CDCl}_{3}\right): \delta$ 22.78-41.06 $\left(\mathrm{CH}_{2}\right) ; 52.95\left(\mathrm{CH}_{3}\right) ; 54.79-55.33(\mathrm{CH}) ; 67.19$ and 67.70 $\left(\mathrm{CH}_{2}-\mathrm{O}\right) ; 128.60-129.11$ (CH arom); 136.81 and 137.33 (C arom); 157.23-157.45 (CO-O$\mathrm{NH}) ; 173.16$ (CO ester); FAB MS: $953.4 \mathrm{~m} / z\left(\mathrm{MH}^{+}\right)$(theory: $\left.952.4 \mathrm{~m} / \mathrm{z}\left(\mathrm{M}^{+}\right)\right)$. Elemental analysis: (theory: C, 64.27; H, 6.77; N, 8.82) found C, 63.98; H, 6.79; N, 8.81. 


\section{Synthesis of LysLys(Lys)OMe 4 HCl}

$\mathrm{Pd} / \mathrm{C}(10 \% \mathrm{w} / \mathrm{w})$ was added to a solution of ZLys(Z)Lys(ZLys(Z))OMe $(1 \mathrm{~g}, 1 \mathrm{mmol})$ in $\mathrm{MeOH}(50 \mathrm{~mL})$. The flask for catalytic hydrogenolysis was evacuated and filled with 50 psi of $\mathrm{H}_{2}$ before shaking for $10 \mathrm{~h}$. The catalyst was removed by filtration and the catalyst was washed with $\mathrm{MeOH}(20 \mathrm{~mL})$. The solution containing the product was acidified with $\mathrm{HCl}$ gas. The acid solution was evaporated to give $578 \mathrm{mg}$ of the white compound (98\%). ${ }^{1} \mathrm{H}$ NMR (DMSO-d ${ }_{6}$ ): $\delta$ 1.36-1.81 (m, 18, $\mathrm{CH}_{2}-\mathrm{CH}_{2}$ ); 2.75 (m, 4, $\mathrm{CH}_{2}-\mathrm{NH}_{3}{ }^{+}$); $3.12\left(\mathrm{~m}, 2, \mathrm{CH}_{2}-\mathrm{NH}\right) ; 3.65$ (s, 3, $\left.\mathrm{CH}_{3}\right) ; 3.82(\mathrm{~m}, 1, \mathrm{CH}-\mathrm{NH}) ; 3.98$ (m, 1, CH-NH); 4.25 $(\mathrm{m}, 1, \mathrm{CH}-\mathrm{NH}) ; 8.20-8.45\left(\mathrm{~m}, 12, \mathrm{NH}_{3}{ }^{+}\right) ; 8.88\left(\mathrm{t}, 1, \mathrm{CH}_{2}-\mathrm{NH}\right) ; 9.18(\mathrm{~d}, 1, \mathrm{CH}-\mathrm{N} H) .{ }^{13} \mathrm{C}$ NMR $\left(\mathrm{CD}_{3} \mathrm{OD}\right): \delta$ 23.40-41.23 $\left(\mathrm{CH}_{2}\right) ; 50.32\left(\mathrm{CH}_{3}\right) ; 53.80-55.10(\mathrm{CH}) ; 170.80-171.18$ (CO-NH); 174.61 (CO ester); FAB MS: $417.4 \mathrm{~m} / \mathrm{z}\left(\mathrm{MH}^{+}-4 \mathrm{HCl}\right)$ (theory: $416.3 \mathrm{~m} / \mathrm{z}$ $\left(\mathrm{M}^{+}\right)$). Elemental analysis: (theory: $\mathrm{C}, 40.65 ; \mathrm{H}, 7.72 ; \mathrm{Cl}, 25.26 ; \mathrm{N}, 14.97$ ) found $\mathrm{C}$, 40.31; H, 7.87; Cl, 25.10; N, 14.97.

\section{Synthesis of isoCys(Boc)Lys(isoCys(Boc))Lys(isoCys(Boc)Lys(isoCys(Boc)))OMe}

LysLys(Lys)OMe.4HCl $(500 \mathrm{mg}, 0.8 \mathrm{mmol})$ was dissolved in DMF $(25 \mathrm{~mL})$, and DIEA $(550 \mathrm{mg}, 4 \mathrm{mmol})$ and HOBT $(695 \mathrm{mg}, 4 \mathrm{mmol})$ were added. After 5 minutes the IsoCys(Boc)OPFP $(2.78 \mathrm{~g}, 5.6 \mathrm{mmol})$ in $\mathrm{CH}_{2} \mathrm{Cl}_{2}(21 \mathrm{~mL})$ was added at $0{ }^{\circ} \mathrm{C}$. The reaction mixture was stirred for $24 \mathrm{~h}$ at RT under $\mathrm{N}_{2}$. After concentration under vacuum the mixture was dissolved in $\mathrm{DCM}(40 \mathrm{~mL})$ and washed with $\mathrm{NaHCO}_{3}(2 \times 100 \mathrm{~mL})$, water $(2 \times 100 \mathrm{~mL})$, and then dried over $\mathrm{Na}_{2} \mathrm{SO}_{4}$. Evaporation of the organic solvent gave an oil that was purified by silica gel chromatography $\left(\mathrm{CH}_{2} \mathrm{Cl}_{2} / \mathrm{MeOH}=96 / 4\right)$ : yield 951 $\mathrm{mg}(96 \%) .{ }^{1} \mathrm{H}$ NMR $\left(\mathrm{CDCl}_{3}\right)$ : $\delta$ 1.19-1.68 (m, 18, $\left.\mathrm{CH}_{2}-\mathrm{CH}_{2}\right) ; 1.43$ (s, 36, C- $\left.\left(\mathrm{CH}_{3}\right)_{3}\right) ; 1.74$ and $1.83\left(2 \times\right.$ s, 24, $\left.\mathrm{C}-\mathrm{CH}_{3}\right) ; 3.22\left(\mathrm{~m}, 14, \mathrm{CH}_{2}-\mathrm{NH}\right.$ and $\left.\mathrm{CH}_{2}-\mathrm{S}\right) ; 3.68$ (s, 3, $\left.\mathrm{CH}_{3}-\mathrm{O}\right) ; 4.29$ (m, 1, CH-NH); 4.40 (m, 1, CH-NH); 4.49 (m, 1, CH-NH); 4.69 (m, 4, CH-N); 6.40-7.00 (m, 6, NH). ${ }^{13} \mathrm{C}$ NMR $\left(\mathrm{CDCl}_{3}\right): \delta$ 22.69-25.47 $\left(\mathrm{CH}_{2}\right) ; 28.96-30.27\left(\mathrm{CH}_{3}\right) ; 31.43\left(\mathrm{CH}_{2}-\mathrm{S}\right)$; $34.32\left(\mathrm{CH}_{2}\right) ; 37.11\left(\mathrm{CH}_{2}\right) ; 39.98\left(\mathrm{CH}_{2}\right) ; 52.74-53.32\left(\mathrm{CO}_{2}-\mathrm{CH}_{3}\right.$ and $\left.\mathrm{CH}\right) ; 67.90(\mathrm{CH})$; 72.00-74-10 $\left(\mathrm{C}^{\left.-\mathrm{CH}_{3}\right)}\right.$; $82.05\left(\mathrm{C}-\left(\mathrm{CH}_{3}\right)_{3}\right) ; 152.32-154.23(\mathrm{O}-\mathrm{CO}-\mathrm{NH}) ; 163.17(\mathrm{CO}-$ $\left.\mathrm{OCH}_{3}\right)$; 171.58-173.07 (CO). FAB MS: $1389.6 \mathrm{~m} / z\left(\mathrm{MH}^{+}\right)$(theory: $1388.6 \mathrm{~m} / \mathrm{z}\left(\mathrm{M}^{+}\right)$). HR MS: $1390.8784 \mathrm{~m} / \mathrm{z}\left(\mathrm{MH}^{+}\right)$(theory: $1390.8799 \mathrm{~m} / \mathrm{z}\left(\mathrm{MH}^{+}\right)$). Elemental analysis: (theory: C, 54.44; H, 7.83; N, 10.08; S, 9.23) found C, 53.93; H, 7.70; N, 9.92; S, 9.15.

\section{Synthesis of isoCysLys(isoCys)Lys(isoCysLys(isoCys)) OMe $4 \mathrm{CF}_{3} \mathrm{CO}_{2} \mathrm{H}$}

TFA $(5 \mathrm{~mL})$ was added in 10 portions over 10 minutes to a solution of isoCys(Boc)Lys(isoCys(Boc))Lys(isoCys(Boc)Lys(isoCys(Boc))) OMe (600 mg, 0.4 mmol) in freshly distilled $\mathrm{CH}_{2} \mathrm{Cl}_{2}(30 \mathrm{~mL})$ at $0{ }^{\circ} \mathrm{C}$. The reaction mixture was stirred under $\mathrm{N}_{2}$ at $25^{\circ} \mathrm{C}$ for $2 \mathrm{~h}$. The solvent was removed by vacuum, and the mixture was precipitated in ether to give a pure white compound $417 \mathrm{mg}(97 \%) .{ }^{1} \mathrm{H}$ NMR $\left(\mathrm{CD}_{3} \mathrm{OD}\right)$ :

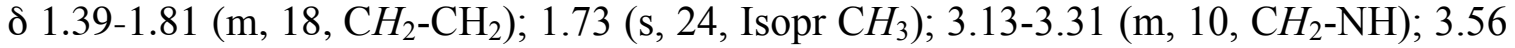
(m, 4, $\mathrm{CH}_{2}-\mathrm{S}$ ); 3.68 (s, 3, $\left.\mathrm{CH}_{3}-\mathrm{O}\right)$ ); 4.27 (m, 1, $\left.\mathrm{CH}-\mathrm{NH}\right) ; 4.36$ (m, 2, $\left.\mathrm{CH}-\mathrm{NH}\right) ; 4.56$ (m, 4, $\mathrm{CH}$-NH-C). ${ }^{13} \mathrm{C}$ NMR $\left(\mathrm{CD}_{3} \mathrm{OD}\right): \delta \quad 24.83-24.96\left(\mathrm{CH}_{2}\right) ; 29.43-30.71\left(\mathrm{CH}_{3}\right) ; 32.87-33.74$ $\left(\mathrm{CH}_{2}-\mathrm{S}\right) ; 36.71-36.95\left(\mathrm{CH}_{2}\right) ; 40.94-41.38\left(\mathrm{CH}_{2}\right) ; 53.68-56.18\left(\mathrm{CH}\right.$ and $\left.\mathrm{CO}_{2}-\mathrm{CH}_{3}\right) ; 65.78$ $(\mathrm{CH})$; 75.55-75.78 (isopr $\mathrm{C})$; $165.31\left(\mathrm{CO}-\mathrm{OCH}_{3}\right) ; 170.17-174.88(\mathrm{CO})$. FAB MS: 990.4 
$m / z\left(\mathrm{MH}^{+}\right)$(theory: $989.4 \mathrm{~m} / z\left(\mathrm{M}^{+}\right)$). Elemental analysis: (theory: $\mathrm{C}, 42.38 ; \mathrm{H}, 5.58 ; \mathrm{N}$, 9.69; S, 8.87) found $\mathrm{C}, 42.10 ; \mathrm{H}, 5.77 ; \mathrm{N}, 9.92 ; \mathrm{S}, 9.01$.

\section{Synthesis of CysLys(Cys)Lys(CysLys(Cys))OMe• 4 HCl (3)}

isoCysLys(isoCys)Lys(isoCysLys(isoCys))OMe (400 $\mathrm{mg}, 0.4 \mathrm{mmol}$ ) was dissolved in $\mathrm{HCl} 1 \mathrm{~N} / \mathrm{MeOH} 50 / 50(60 \mathrm{~mL})$ and stirred under $\mathrm{N}_{2}$ at $25{ }^{\circ} \mathrm{C}$ for $4 \mathrm{~h}$. The solvent was removed by vacuum, and the mixture was precipitated in ether to give a pure white compound $350 \mathrm{mg}(90 \%)$. ${ }^{1} \mathrm{H}$ NMR (DMSO d-6): $\delta 1.30-1.76\left(\mathrm{~m}, 18, \mathrm{CH}_{2}-\mathrm{CH}_{2}\right) ; 2.76-$ 3.19 (m, 18, $\mathrm{CH}_{2}-\mathrm{NH}, \mathrm{CH}_{2}-\mathrm{SH}$ and $\left.\mathrm{CH}_{2}-\mathrm{SH}\right) ; 3.63$ (s, 3, $\left.\mathrm{CH}_{3}-\mathrm{O}\right) ; 4.02$ (m, 2, $\mathrm{CH}_{-}-\mathrm{NH}_{3}{ }^{+}$); $4.13\left(\mathrm{~m}, 2, \mathrm{CH}-\mathrm{NH}_{3}{ }^{+}\right) ; 4.18(\mathrm{~m}, 2, \mathrm{CH}-\mathrm{NH}) ; 4.32$ (m, 1, NH-CH-CO$\left.{ }_{2} \mathrm{CH}_{3}\right) ; 8.18,8.47$ and $8.81\left(\mathrm{~m}, 18, \mathrm{NH}\right.$ and $\left.\mathrm{NH}_{3}{ }^{+}\right) .{ }^{13} \mathrm{C}$ NMR (DMSO d- $\left.{ }_{6}\right): \delta$ 23.34-25.87 $\left(\mathrm{CH}_{2}\right) ; 28.91$ $\left(\mathrm{CH}_{2}\right) ; 31.99\left(\mathrm{CH}_{2}\right) ; 49.23\left(\mathrm{CH}_{2}-\mathrm{SH}\right) ; 52.49\left(\mathrm{CO}_{2}-\mathrm{CH}_{3}\right) ; 52.49-54.56(\mathrm{CH}) ; 167.36(\mathrm{CO}-$ $\left.\mathrm{OCH}_{3}\right)$; 172.26-178.39 (CO). FAB MS: $829.6 \mathrm{~m} / z\left(\mathrm{MH}^{+}\right)$(theory: $\left.828.3 \mathrm{~m} / \mathrm{z}\left(\mathrm{M}^{+}\right)\right)$. HR MS: $829.3581 \mathrm{~m} / z\left(\mathrm{MH}^{+}\right)$(theory: $\left.828.3478 \mathrm{~m} / z\left(\mathrm{M}^{+}\right)\right)$. Elemental analysis: (theory: $\mathrm{C}$, $38.19 ; \mathrm{H}, 6.62 ; \mathrm{N}, 14.37)$ found: $\mathrm{C}, 37.99 ; \mathrm{H}, 6.67 ; \mathrm{N}, 14.21$.

\section{Synthesis of BocLys(Boc)OPFP}

BocLys(Boc)OPFP was prepared in a similar manner as ZLys(Z)OPFP starting from BocLys(Boc)OH in 92\% yield. ${ }^{1} \mathrm{H}$ NMR $\left(\mathrm{CDCl}_{3}\right): \delta 1.43\left(\mathrm{~s}, 18, \mathrm{Boc} \mathrm{CH}_{3}\right) ; 1.52(\mathrm{~m}, 2$, $\left.\mathrm{CH}_{2}\right) ; 1.87\left(\mathrm{~m}, 2, \mathrm{CH}_{2}\right) ; 1.95\left(\mathrm{~m}, 2, \mathrm{CH}_{2}\right) ; 3.12\left(\mathrm{~m}, 2, \mathrm{CH}_{2}-\mathrm{NH}\right)$ ); 4.57 (m, 1, CH); 5.24 $(\mathrm{m}, 1, \mathrm{NH}) .{ }^{13} \mathrm{C} \mathrm{NMR}\left(\mathrm{CDCl}_{3}\right): \delta 22.23\left(\mathrm{CH}_{2}\right) ; 28.33\left(\mathrm{CH}_{3}\right) ; 29.21-31.12\left(\mathrm{CH}_{2}\right) ; 39.70$ $\left(\mathrm{CH}_{2} \mathrm{NH}\right) ; 53.53(\mathrm{CH}) ; 82.50$ and $83.13\left(\mathrm{C}-\left(\mathrm{CH}_{3}\right)_{3}\right) ; 157.2-157.5(\mathrm{CONH}) ; 169.46(\mathrm{CO}$ ester). ${ }^{19} \mathrm{~F}$ NMR $\left(\mathrm{CDCl}_{3}\right)$ : $\delta-162.22(\mathrm{t}, 2, \mathrm{CF}) ;-157.72$ (t, 1, CF); -152.97 (d, 2, CF). FAB MS: $535.7 \mathrm{~m} / \mathrm{z}\left(\mathrm{M}+\mathrm{Na}^{+}\right)$(theory: $\left.512.4 \mathrm{~m} / z\left(\mathrm{M}^{+}\right)\right)$. Elemental analysis: (theory: $\mathrm{C}$, $51.56 ; \mathrm{H}, 5.70 ; \mathrm{N}, 5.47)$ found $\mathrm{C}, 51.49 ; \mathrm{H}, 5.68 ; \mathrm{N}, 5.41$.

\section{Synthesis of BocLys(Boc)Lys(BocLys(Boc))Lys(BocLys(Boc)Lys(BocLys(Boc)))OMe BocLys(Boc)Lys(BocLys(Boc))Lys(BocLys(Boc)Lys(BocLys(Boc)))OMe was prepared in a similar manner as ZLys(Z)Lys(ZLys(Z))OMe but starting from LysLys(Lys)OMe $4 \mathrm{HCl}$. LysLys(Lys)OMe $4 \mathrm{HCl}(300 \mathrm{mg}, 0.53 \mathrm{mmol})$ was dissolved in DMF (45 mL) and DIEA (330 mg, $2.5 \mathrm{mmol})$, HOBT (390 $\mathrm{mg}, 2.5 \mathrm{mmol})$ and (Boc)Lys(Boc)OPFP (1.6 g, $3.2 \mathrm{mmol}$ ) were added to the solution. The reaction was run for $24 \mathrm{~h}$ at RT. The solution was concentrated and the product purified by silica gel chromatography $\left(\mathrm{CH}_{2} \mathrm{Cl}_{2} / \mathrm{MeOH}=95 / 5\right)$ : yield $600 \mathrm{mg}(65 \%) .{ }^{1} \mathrm{H}$ NMR (DMSO d- -6$): \delta$ 1.16-1.62 (m, 42, $\left.\mathrm{CH}_{2}-\mathrm{CH}_{2}\right) ; 1.32$ (s, 72, Boc $\left.\mathrm{CH}_{3}\right) ; 2.81$ (m, 8, $\mathrm{CH}_{2}-\mathrm{NH}$ Boc); 2.96 (m, 6, $\left.\mathrm{CH}_{2}-\mathrm{NH}\right) ; 3.56$ (s, 3, $\left.\mathrm{CH}_{3}-\mathrm{O}\right) ; 3.76$ (m, 4, $\mathrm{CH}-\mathrm{NH}$ Boc); 4.12 (m, 2, CH-NH); 4.25 (m, 1, NH-CH$\left.-\mathrm{CO}_{2} \mathrm{CH}_{3}\right) ; 6.43,6.67,6.85,7.69,7.84$ and $8.24(\mathrm{~m}, 14, \mathrm{NH})$. FAB MS: 1730.7 $\mathrm{m} / z\left(\mathrm{MH}^{+}\right)$(theory: $\left.1729.1 \mathrm{~m} / z\left(\mathrm{M}^{+}\right)\right) .{ }^{13} \mathrm{C}$ NMR (DMSO d- $): \delta 28.10\left(\mathrm{CH}_{2}\right) ; 33.41-$ $37.12\left(\mathrm{CH}_{2}\right.$ and $\left.\mathrm{CH}_{3}\right) ; 52.80\left(\mathrm{OCH}_{3}\right) ; 56.95(\mathrm{CH}) ; 59.59(\mathrm{CH}) ; 82.53-83.13\left(C-\left(\mathrm{CH}_{3}\right)_{3}\right)$; 157.23-158.10 (CONH); 160.75 ( $C \mathrm{O}$ ester); 177.05-177.51 (CO-NH). Elemental analysis: (theory: $\mathrm{C}, 57.62 ; \mathrm{H}, 8.85 ; \mathrm{N}, 11.33$ ) found $\mathrm{C}, 57.37 ; \mathrm{H}, 8.94 ; \mathrm{N}, 11.10$.}

\section{Synthesis of LysLys(Lys)Lys(LysLys(Lys))OMe• $8 \mathrm{CF}_{3} \mathrm{CO}_{2} \mathrm{H}$}


LysLys(Lys)Lys(LysLys(Lys))OMe was prepared in a similar manner as above for is o Cys Lys (iso Cys) Lys but starting from BocLys(Boc)Lys(BocLys(Boc))Lys(BocLys(Boc)Lys(BocLys(Boc)))OMe was dissolved in $\mathrm{CH}_{2} \mathrm{Cl}_{2}$ and TFA was added. The reaction was run for $1 \mathrm{~h}$ at RT. The product was isolated after evaporation of the solvent $(100 \mathrm{mg}, 0.057 \mathrm{mmol})(99 \%) .{ }^{1} \mathrm{H}$ NMR (DMSO d-6): $\delta$ 1.26-1.64 (m, 48, $\left.\mathrm{CH}_{2}-\mathrm{CH}_{2}\right) ; 2.76\left(\mathrm{~m}, 8, \mathrm{CH}_{2}-\mathrm{NH}\right) ; 3.04$ (m, 6, $\left.\mathrm{CH}_{2}-\mathrm{NH}\right) ; 3.57$ (s, 3, $\left.\mathrm{CH}_{3}\right) ; 3.65$ and $3.76\left(\mathrm{~m}, 4, \mathrm{CH}-\mathrm{NH}_{3}{ }^{+}\right) ; 4.12(\mathrm{~m}, 2, \mathrm{CH}-\mathrm{NH}) ; 4.25$ (m, 4, NH-CH$\left.\mathrm{CO}_{2} \mathrm{CH}_{3}\right) .7 .82,8.15$, and $8.50\left(\mathrm{~m}, 30, \mathrm{NH}\right.$ and $\mathrm{NH}_{3}{ }^{+}$). ${ }^{13 \mathrm{C}} \mathrm{NMR}$ (DMSO d-6): $\delta 21.12-$ $31.25\left(\mathrm{CH}_{2}\right) ; 52.53-52.77\left(\mathrm{CH}\right.$ and $\left.\mathrm{CH}_{3}\right) ; 159.74$ ( $\mathrm{CO}$ ester); 168.80-173.04 (CONH).FAB MS: $929.2 \mathrm{~m} / \mathrm{z}\left(\mathrm{MH}^{+}\right)$(theory: $928.7 \mathrm{~m} / \mathrm{z}\left(\mathrm{M}^{+}\right)$). Elemental analysis: (theory: C, 55.58; H, 9.55; N, 21.10) found $\mathrm{C}, 55.30 ; \mathrm{H}, 9.59 ; \mathrm{N}, 21.00$.

\section{Synthesis of (isoCys(Boc) $)_{8}(\mathrm{Lys})_{7} \mathrm{OMe}$}

LysLys(Lys)Lys(LysLys(Lys))OMe $\bullet 8 \mathrm{CF}_{3} \mathrm{CO}_{2} \mathrm{H}(106 \mathrm{mg}, 0.057 \mathrm{mmol})$ was dissolved in DMF $(10 \mathrm{~mL})$, and DIEA $(71 \mathrm{mg}, 0.55 \mathrm{mmol})$ and HOBT $(90 \mathrm{mg}, 0.55 \mathrm{mmol})$ were added. After $5 \mathrm{~min}$ the IsoCys(Boc)OPFP (300 $\mathrm{mg}, 0.7 \mathrm{mmol})$ in $\mathrm{CH}_{2} \mathrm{Cl}_{2}(10 \mathrm{~mL})$ was added at $0{ }^{\circ} \mathrm{C}$. The reaction mixture was stirred for $24 \mathrm{~h}$ at RT under $\mathrm{N}_{2}$. After concentration under vacuum the mixture was dissolved in DCM $(40 \mathrm{~mL})$ and washed with $\mathrm{NaHCO}_{3}(2 \times 100 \mathrm{~mL})$, water $(2 \times 100 \mathrm{~mL})$, and then dried over $\mathrm{Na}_{2} \mathrm{SO}_{4}$. Evaporation of the organic solvent gave an oil that was purified using Sephadex LH20 $\left(\mathrm{CH}_{2} \mathrm{Cl}_{2} / \mathrm{MeOH}=50 / 50\right)$ : yield $160 \mathrm{mg}(96 \%)$. ${ }^{1} \mathrm{H}$ NMR (DMSO d-6): $\delta$ 1.16-1.71 (m, 42, $\mathrm{CH}_{2}-\mathrm{CH}_{2}$ ); 1.27 (s, 81, Boc $\mathrm{CH}_{3}$ ); 1.65 and 1.71 (s, 48, Isopr $\left.\mathrm{CH}_{3}\right) ; 2.85-3.29$ (m, 30, $\mathrm{CH}_{2}-\mathrm{NH}$ and $\left.\mathrm{CH}_{2}-\mathrm{S}\right) ; 3.56$ (s, 3, $\left.\mathrm{CH}_{3}-\mathrm{O}\right) ; 4.11-4.63$ (m, 15, $\left.\mathrm{CH}-\mathrm{NH}\right) ; 7.62-7.93$ (m, 14, $\mathrm{NH}) .{ }^{3} \mathrm{C}$ NMR (DMSO d- 6 ): $\delta 23.13\left(\mathrm{CH}_{2}\right) ; 28.48-36.87\left(\mathrm{CH}_{2}, \mathrm{CH}_{3}\right.$, and $\left.\mathrm{CH}_{2}-\mathrm{S}\right) ; 52.50-$ $52.92(\mathrm{CH}) ; 53.12\left(\mathrm{OCH}_{3}\right) ; 67.02(\mathrm{CH}) ; 71.80-71.99\left(\mathrm{C}_{-} \mathrm{CH}_{3}\right) ; 80.06-81.10\left(\mathrm{C}-\left(\mathrm{CH}_{3}\right)_{3}\right)$; 152.22-152.32 (CONH); 160.21 (CO ester); 170.68-171.81 (CO-NH). MALDI: $2898 \mathrm{~m} / \mathrm{z}$ $\left(\mathrm{M}+\mathrm{Na}^{+}\right)$(theory: $2875 \mathrm{~m} / z\left(\mathrm{M}^{+}\right)$). Elemental analysis: (theory: $\mathrm{C}, 54.71 ; \mathrm{H}, 7.85 ; \mathrm{N}$, 10.72) found $\mathrm{C}, 54.33 ; \mathrm{H}, 7.95 ; \mathrm{N}, 10.45$.

\section{Synthesis of (isoCys) ${ }_{8}(\mathrm{Lys})_{7} \mathrm{OMe} \bullet \mathrm{8CF}_{3} \mathrm{CO}_{2} \mathrm{H}$}

TFA (3 $\mathrm{mL})$ was added in 10 portions over $10 \mathrm{~min}$ to a solution of (isoCys(Boc) $)_{8}(\mathrm{Lys})_{7} \mathrm{OMe}(480 \mathrm{mg}, 0.16 \mathrm{mmol})$ in freshly distilled $\mathrm{CH}_{2} \mathrm{Cl}_{2}(21 \mathrm{~mL})$ at 0 ${ }^{\circ} \mathrm{C}$. The reaction mixture was stirred under $\mathrm{N}_{2}$ at $25{ }^{\circ} \mathrm{C}$ for $2 \mathrm{~h}$. The solvent was removed by vacuum, and the mixture was precipitated in ether to give a pure white compound 480 mg (97 \%). ${ }^{1} \mathrm{H}$ NMR (DMSO d-6): $\delta$ 1.27-1.68 (m, 42, $\mathrm{CH}_{2}-\mathrm{CH}_{2}$ ); 1.65 (s, 48, Isopr $\left.\mathrm{CH}_{3}\right) ; 3.00$ (m, 8, $\left.\mathrm{CH}_{2}-\mathrm{S}\right) ; 3.13$ (m, 14, $\left.\mathrm{CH}_{2}-\mathrm{NH}\right) ; 3.50$ (m, 8, $\left.\mathrm{CH}_{2}-\mathrm{S}\right) ; 3.60$ (s, 3, $\mathrm{CH}_{3}-\mathrm{O}$ ); 4.15-4.41 (m, 15, $\mathrm{CH}-\mathrm{NH}) ; 8.04-8.47(\mathrm{~m}, 22, \mathrm{NH}) .{ }^{13} \mathrm{C} \mathrm{NMR}\left(\mathrm{CD}_{3} \mathrm{OD}\right): \delta 22.62\left(\mathrm{CH}_{2}\right)$; 27.63-33.84 $\left(\mathrm{CH}_{2}, \mathrm{CH}_{3}\right.$, and $\left.\mathrm{CH}_{2}-\mathrm{S}\right) ; 50.92-52.98\left(\mathrm{CH}\right.$ and $\left.\mathrm{OCH}_{3}\right) ; 62.85(\mathrm{CH}) ; 72.21(\mathrm{C}-$ $\left.\mathrm{CH}_{3}\right) ; 166.21$ (CO ester); 170.60-171.30 (CO-NH). MALDI: $2074 \mathrm{~m} / \mathrm{z}\left(\mathrm{MH}^{+}\right)$(theory: $2073 \mathrm{~m} / \mathrm{z}\left(\mathrm{M}^{+}\right)$). Elemental analysis: (theory: $\mathrm{C}, 39.50 ; \mathrm{H}, 4.90 ; \mathrm{N}, 8.66$ ) found $\mathrm{C}, 39.30$; $\mathrm{H}, 5.00 ; \mathrm{N}, 8.45$.

\section{Synthesis of (Cys) $)_{8}(\mathrm{Lys})_{7} \mathrm{OMe} \cdot 8 \mathrm{HCl}(4)$}

(isoCys) $)_{8}(\mathrm{Lys})_{7} \mathrm{OMe} \bullet 8 \mathrm{CF}_{3} \mathrm{CO}_{2} \mathrm{H}(480 \mathrm{mg}, 0.16 \mathrm{mmol})$ was dissolved in $\mathrm{HCl} 1 \mathrm{~N} / \mathrm{MeOH}$ $50 / 50(150 \mathrm{~mL})$ and stirred under $\mathrm{N}_{2}$ at $25{ }^{\circ} \mathrm{C}$ for $4 \mathrm{~h}$. The solvent was removed by 
vacuum, and the mixture was precipitated in ether to give a pure white compound $467 \mathrm{mg}$ (99 \%). ${ }^{1} \mathrm{H}$ NMR (DMSO d-6): $\delta$ 1.18-1.68 (m, 42, $\mathrm{CH}_{2}-\mathrm{CH}_{2}$ ); 2.69-3.11 (m, 38, $\mathrm{CH}_{2-}$ $\mathrm{NH}, \mathrm{CH}_{2}-\mathrm{SH}$ and $\left.\mathrm{CH}_{2}-\mathrm{SH}\right) ; 3.55$ (s, 3, $\left.\mathrm{CH}_{3}-\mathrm{O}\right)$; 4.88-4.22 (m, 15, CH-NH${ }_{3}^{+}$and $\mathrm{CH}-$ $\mathrm{NH}) ; 8.16,8.35$ and $8.74\left(\mathrm{~m}, 38, \mathrm{NH}\right.$ and $\mathrm{NH}_{3}{ }^{+}$). ${ }^{13} \mathrm{C}$ NMR (DMSO d-6): $\delta$ 23.10-31.80 $\left(\mathrm{CH}_{2}\right) ; 49.12\left(\mathrm{CH}_{2}-\mathrm{S}\right) ; 52.40-54.30\left(\mathrm{CH}_{3}\right.$ and $\left.\mathrm{CH}\right) ; 167.28$ (CO ester); 172.20-178.31 (CO-NH). MALDI: $1777.1 \mathrm{~m} / z\left(\mathrm{M}+\mathrm{Na}^{+}\right)$(theory: $\left.1754.4 \mathrm{~m} / z\left(\mathrm{M}^{+}\right)\right)$. Elemental analysis: (theory: C, 36.11; H, 6.38; N, 13.83) found C, 36.01; H, 6.46; N, 13.03.

\section{Mechanical Properties:}

The rheological measurements were obtained on a TA Instruments RA 1000 instrument. To prepare the hydrogels, dendron $\mathbf{3}$ or $\mathbf{4}$ was reacted with poly(ethylene glycol dialdehyde) of $3400 \mathrm{Mw}$ (5, PEG-DA) in HEPES buffer at $\mathrm{pH}=7.4$. The ratio of cysteine to aldehyde was $1: 1$, and the total concentration of polymer in solution was either 30 or $50 \% \mathrm{w} / \mathrm{w}$. A hydophilic gel formed spontaneously within three minutes upon mixing the two aqueous solutions at either concentration. Cylindrical hydrogel samples of $8 \mathrm{~mm}$ diameter and $2 \mathrm{~mm}$ thickness were prepared in a precast teflon mold and analyzed after setting at $25{ }^{\circ} \mathrm{C}$ for 24 hours. The mechanical properties were measured at a frequency of 0.1 to $10 \mathrm{~Hz}$ with a controlled strain of $0.4 \%$ and at $25{ }^{\circ} \mathrm{C}$. A normal force of $0.4 \mathrm{~N}$ was applied to the gel using an $8 \mathrm{~mm}$ steel plate geometry. Data are reported at a frequency of $1 \mathrm{~Hz}$. The gels exhibited viscoelastic properties. The complex modulus $\left(\mathrm{G}^{*}\right)$ for the $30 \% \mathrm{w} / \mathrm{w}$ hydrogels prepared from $\mathbf{3}$ or $\mathbf{4}$ and $\mathbf{5}$ was $3.8 \mathrm{x}$ $10^{3}$ and $1.0 \times 10^{4}$, respectively. The increase in modulus is consistent with the increase in crosslinking density present in the $\mathbf{4 \cdot 5}$ hydrogel. Hydrogel properties are highly dependent on dendron generation, $\% \mathrm{w} / \mathrm{w}$, and macromer stoichiometry, and thus we focused our initial study on these formulations which sealed a clear corneal laceration.

\section{Clear Corneal Incision:}

An enucleated human eye (NC Eye Bank) was secured under a surgical microscope with the cornea facing upwards. A cardiac transducer (Hewlett-Packard, Palo Alto, CA) was primed and a 20-gauge needle (Sherwood Medical, St. Louis, MO) was attached to the end of the saline tubing leading from the transducer. This needle was inserted into the optic nerve approximately $1 \mathrm{~cm}$ into the globe. It is not necessary to tie the needle and optic nerve together in order to secure the needle. Eye Bank eyes with little to no optic nerve were not used. The age and overall quality of the eye bank eyes varied. Next, a 24-gauge butterfly needle connected to a saline filled 10-cc syringe (Becton Dickinson \& Co., Rutherford, NJ) in a syringe pump (KdScientific Model 100) was inserted into the anterior chamber through the peripheral cornea. A $3.0 \mathrm{~mm}$ dual beveled, angled slit knife (Alcon, Ft. Worth, TX) was used to make a $3.0 \mathrm{~mm}$ clear corneal linear incision (perpendicular to the plane of the cornea) 90 degrees from the orientation of the butterfly needle. The cardiac monitor was set to an arterial pressure setting and adjusted to zero $\mathrm{mm} \mathrm{Hg}$. The wound was wiped with an Opticel sponge to dry the wound. If desired, the edges of the wound can be marked with a pen such as a Devon Skin Marker and Ruler, Regular tip \#150 (Tyco Healthcare, Japan). The macromer solution was mixed and applied $(5-10 \mu \mathrm{L})$ to the dried wound. A hydrogel 
patch formed within a few minutes. For the suture group, one interrupted 10-0 nylon suture was used to close the $3.0 \mathrm{~mm}$ clear corneal linear incision using a needle holder and 0.12 forceps. The self-seal wound group was not repaired. Fluoroscein dye was applied to the wound and the surrounding area using Fluorets strip (Chavvin) to look for wound leakage. Within 5 minutes of the repairing the wound, saline was injected into the eye using the syringe pump at a rate of $8 \mathrm{~mL} /$ hour to increase the IOP as measured by the transducer. The cardiac transducer monitored the IOP of the repaired eyes as we have done previously. A Tonopen (Medtronic Solan, Jacksonville, FL) was used to confirm the concordance of the transducer readings at low pressure readings. We checked for signs of leakage and recorded the IOP reading from the transducer when leaking was observed at the wound or around the wound.

The incision is not sealed using only the dendron or PEG-DA hydrogel precursors. The hydrogel sealant secures the clear corneal incision and withstands higher pressures and stress placed on a wound than conventional suture or self-sealed treated wounds. Compared to the self-sealing group the hydrogel sealant maintains a closed wound at low and high intraocular

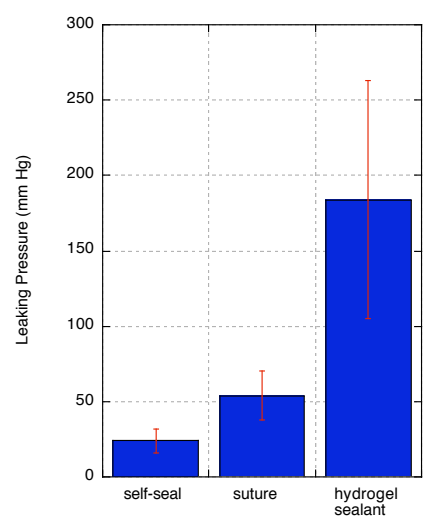

Table S1. Leaking pressures for clear corneal incisions that were "self-sealed", sutured, or sealed with the hydrogel.

pressures. The procedure with the hydrogel sealant is facile and requires less surgical time than conventional suturing (4 to 6 times) and does not inflict additional tissue trauma. 